Classification

Physics Abstracts

42.30va

\title{
Microscope à double détection : STOM-AFM, principe et résultats
}

\author{
Fadi Baida, Chekib Mered $\left(^{*}\right)$ et Daniel Courjon \\ Laboratoire d'Optique P.M. Duffieux, URA 214 CNRS, Université de Franche-Comté, UFR Sciences, \\ Route de Gray 25030 Besançon Cedex, France
}

(Reçu le 6 octobre 1993, accepté le 19 octobre 1993)

\begin{abstract}
Résumé. - Le microscope optique en champ proche (SNOM) fournit une cartographie haute résolution de l'intensité lumineuse au voisinage immédiat de la surface de l'objet. Cette intensité est corrélée à la fois au profil géométrique de la surface et à la nature physico-chimique des matériaux constituant l'objet à imager [1]. Afin de décorréler même partiellement ces informations, un microscope hybride a été réalisé. Il combine une détection AFM (Atomic Force Microscope) avec une détection STOM (Scanning Tunneling Optical Microscope), variante technique du microscope optique en champ proche. Les informations peuvent être obtenues simultanément ou séquentiellement sur le même site d'exploration. A la suite du descriptif technique du travail effectué, nous conclurons par la présentation des premiers résultats.
\end{abstract}

\begin{abstract}
Near field optical microscopy provides a high resolved map of the light intensity very close to the object surface. This intensity is directly correlated with the profile and with the physicochemical nature of the material constituting the object. A hybrid microscope has been built to partially decorrelate these data. It combines an AFM (Atomic Force Microscopy) detection with a STOM (Scanning Tunneling Optical Microscopy) detection. The information can be obtained simultaneously or sequentially on the same scanned area. After a technical description of the realized device, we will conclude by presenting the first results.
\end{abstract}

\section{Introduction.}

La microscopie optique en champ proche est basée sur la détection des ondes évanescentes qui contiennent les informations relatives aux détails sub-longueur d'onde de la surface [2]. Ces ondes se propagent parallèlement à la surface et leur intensité décroît exponentiellement lorsque le plan d'analyse s'éloigne de cette dernière. Pour les détecter, il est nécessaire que la sonde soit placée au voisinage immédiat de l'objet (c.à.d. à quelques nanomètres).

A la différence de la microscopie optique classique, aucun plan d'analyse ne peut être défini en microscopie en champ proche. Cette particularité propre à toute microscopie à sonde locale

(*) Actuellement à l’Université de TLEMCEM (Algérie). 
interdit la définition d'un critère de résolution standard. En effet, les images obtenues au moyen de deux microscopes identiques peuvent présenter des différences notables, cela étant dû au grand nombre de paramètres définissant les conditions initiales : forme et nature de la pointe, distance d'analyse, etc. Concernant la détection, comme pour toute microscopie à sonde locale, deux modes de fonctionnement ont été développés : le premier, dit à altitude constante, consiste à déplacer la sonde parallèlement à elle-même à une distance constante de la surface moyenne de l'objet, le signal utile dans ce cas est l'intensité lumineuse $I(x, y)$, détectée en chaque point. Le deuxième mode, appelé mode à intensité constante, consiste à asservir, par voie électronique, la position de la sonde pour qu'elle suive une courbe d'iso-intensité lumineuse au-dessus de la surface de l'objet. Le signal utile dans ce cas est la position de la sonde en chaque point $z(x, y)$.

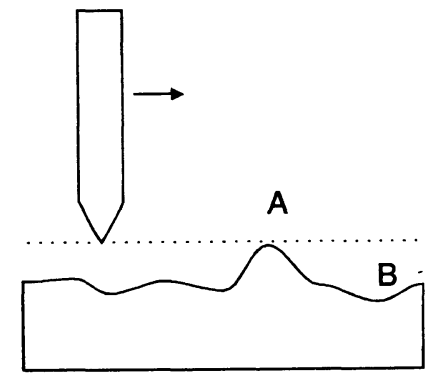

a)

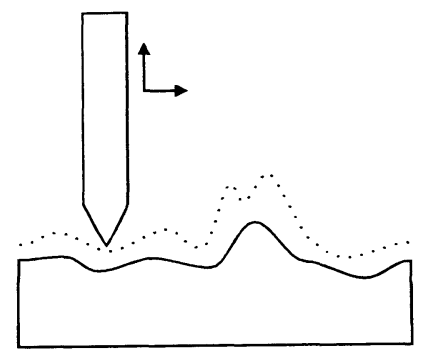

b)

Fig. 1. - Modes de détection dits à altitude constante a) et à intensité constante b).

[The two possible modes of $x y z$ scanning in STOM. Constant altitude mode a) and constant intensity mode b).]

Ces deux modes de détection présentent chacun leurs inconvénients propres : dans le cas du mode à altitude constante (Fig. 1a), il est nécessaire de travailler à une distance suffisante de la surface afin d'éviter l'entrée en contact avec les parties hautes de l'objet. Il est alors clair que des régions de l'image (zone A de la Fig. 1a) vont présenter une bonne résolution (car imagées en champ proche), alors que d'autres régions (zone B de la même figure) seront imagées en champ lointain et présenteront une résolution faible. Cette technique s'appliquera donc à des échantillons à faible relief et dont le plan moyen sera ajusté de telle sorte qu'il soit parallèle au plan de balayage.

Pour le mode à intensité constante (Fig.1b) les risques de contact ne sont pas totalement supprimés. Par exemple, une zone opaque pourra provoquer le déplacement de la sonde vers la surface afin de retrouver la valeur de consigne. Si celle-ci n'est pas atteinte, la pointe poursuivra sa descente et se brisera sur l'objet. Le même risque peut également apparaître avec une inhomogénéité d'indice se traduisant par une variation du champ lumineux à la détection. Celle-ci sera interprétée comme une variation dans le profil géométrique de l'objet. On constate ici, qu'à la différence du STM, l'utilisation d'un asservissement sur le signal lui-même, ne permet pas de maintenir la sonde à une distance constante de l'échantillon. Une exception peut être mentionnée pour les échantillons à faible relief comme il l'a été montré dans la référence [3].

Pour résoudre même partiellement ce problème, quelques groupes [4-6] ont récemment proposé des solutions visant à maintenir la distance objet-pointe constante en utilisant un paramètre annexe (force de friction ou de répulsion) pour assurer le contrôle de distance. 


\section{Microscope hybride AFM $\times$ STOM.}

Le but est de combiner un microscope à mesure de force avec un microscope en champ proche optique et d'asservir la position de la pointe à l'aide du signal topographique issu du microscope à force [4-6]. Il est à craindre, dans ce cas, que le signal détecté soit le résultat de l'interaction entre les phénomènes optiques et les phénomènes de force. Néanmoins, les premiers résultats obtenus, malgré ces réserves, montrent la potentialité de ces techniques hybrides. Dans la variante décrite ici, la pointe est en contact avec l'objet, le signal détecté est alors le champ lumineux sur la surface de l'objet.

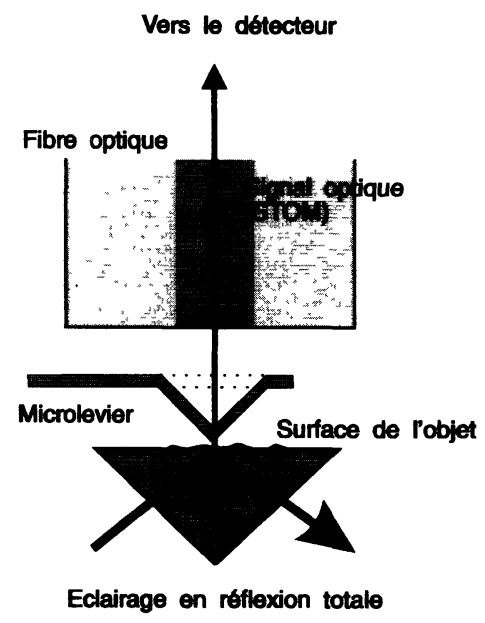

a)

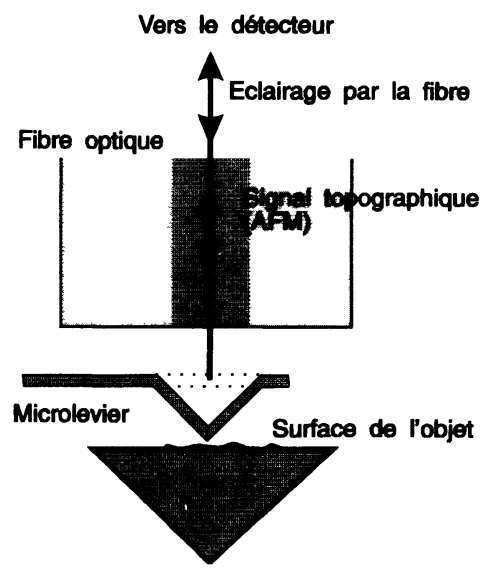

b)

Fig. 2. - Schéma de principe des détections pour le microscope hybride. a) STOM, b) topographique. [Schematic diagram of the hybrid microscope a): STOM, b): topographic.]

2.1 PRINCIPE. - Il s'agit de combiner dans la même tête une détection optique et une détection AFM. Le nanocollecteur est une pointe AFM standard en nitrure de silicium associée à une fibre optique monomode. Le microscope en champ proche optique est décrit sur la figure $2 a$. Un faisceau laser modulé sinusoïdalement en amplitude à une fréquence de quelques $\mathrm{kHz}$ est réfléchi totalement à l'intérieur du prisme. L'onde évanescente générée par cette réflexion totale éclaire l'échantillon. Cette configuration est connue sous le nom de STOM (Scanning Tunneling Optical Microscope) ou PSTM (Photon Scanning Tunneling Microscope). Le champ proche, résultant de l'interaction entre cette onde évanescente et l'objet, est collecté par la pointe AFM. Notons que ce champ est essentiellement constitué de composantes évanescentes. Cette détection s'apparente à une frustration optique c'est-à-dire à la conversion des ondes évanescentes en ondes progressives aptes à se propager à travers la pointe elle-même jusqu'à la face clivée de la fibre, puis de celle-ci au détecteur. Un deuxième faisceau laser modulé en amplitude à une fréquence différente, est injecté dans cette même fibre (voir Fig. 2b). La face clivée de cette dernière et la surface polie du microlevier jouent le rôle d'un interféromètre de Fabry-Pérot pour les différents faisceaux transitant dans la fibre. La phase associée aux franges résultant du phénomène d'interférence 
permettra de déduire la position relative de la pointe par rapport à un plan quelconque initialement défini. Une détection synchrone est alors effectuée permettant de discriminer chacun des signaux. La principale différence entre les techniques déjà développées [5] et celle-ci est liée au fait que la pointe est en contact avec l'objet (AFM en mode répulsif). Il s'agit donc de la détection du champ très proche dont les variations sont corrélées aux propriétés physico-chimiques du matériau. Notons que cette configuration devrait être relativement insensible aux artefacts dus à la propagation du champ puisque cette propagation est fortement minimisée dans ce cas. Les calculs théoriques et les résultats obtenus à l'aide des techniques similaires dans d'autres groupes ont confirmé ces hypothèses [4].

2.2 ETUDE THÉORIQUE. - Par souci de simplicité nous appellerons dans la suite "signal STOM" l'intensité associée au champ proche sur l'objet et "signal topographique" le signal permettant de déterminer la topographie de l'objet.

Il s'agit donc tout d'abord de déterminer les dépendances des intensités respectives des deux signaux en fonction de l'altitude de la pointe (coordonnées $z$ ). L'étude repose sur l'application d'une méthode macroscopique [7] nous permettant de déterminer la transmission d'un ensemble multi-couches.

Les paramètres utilisés dans le calcul sont les suivants :

- indice optique du nitrure de silicium : $2,1+0,00063 i[8]$

- indice de la fibre : 1,46

- indice de l'objet : 1,5 (verre optique)

- épaisseur du microlevier : $646 \mathrm{~nm}$

- épaisseur de la couche métallique (or) : $100 \mathrm{~nm}$

- indice de l'or : $0,21+3,272 i$ [9]

La simulation théorique a été effectuée en deux étapes (Fig. 3) :
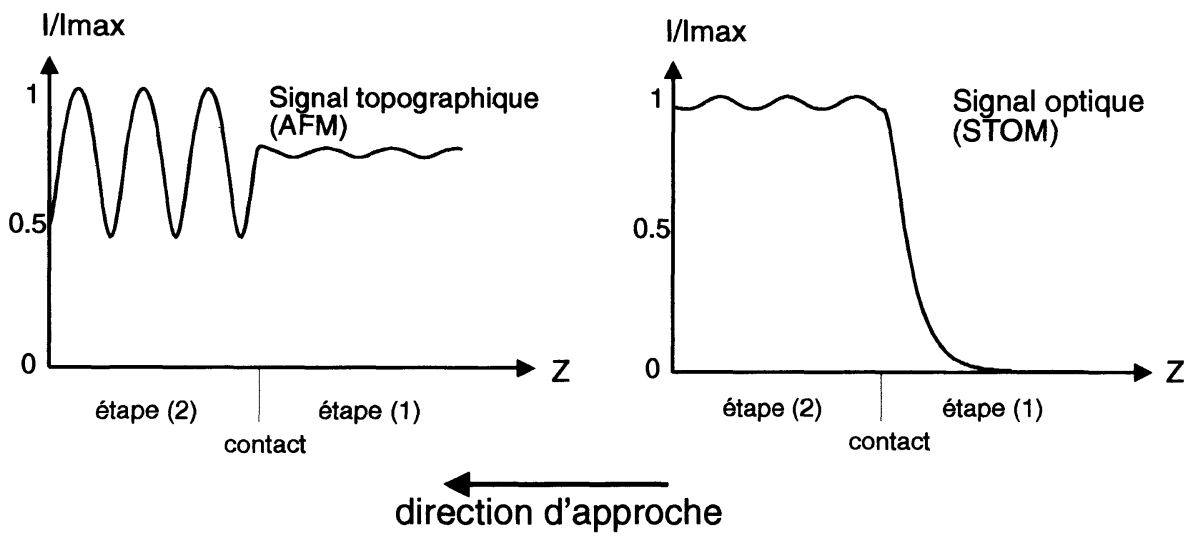

Fig. 3. - Courbes théoriques de l'approche pointe - objet.

[Theoretical approach curves.]

Etape 1 : L'objet situé loin du microlevier (à quelques longueurs d'ondes), s'approche à une vitesse constante de ce dernier. Dans le cas idéal, le microlevier étant théoriquement parfaitement réfléchissant, aucun phénomène d'interférence ne doit apparaître (le microlevier et la fibre sont en effet immobiles). Toutefois, pratiquement, la couche métallique est mince (environ $100 \mathrm{~nm}$ ) afin 
de conserver la faible raideur du microlevier. Le faisceau de contrôle est partiellemnet transmis à travers ce dernier, il est donc réfléchi par l'objet et conduit à un phénomène d'interférence parasite à faible visibilité. Il n'affectera que le signal topographique car la pointe AFM n'a pas encore atteint la zone d'existence de l'onde évanescente confinée à moins d'une demi-longueur d'onde de la surface de l'objet. Le signal STOM est donc nul (voir Fig. 3).

Lorsque la distance entre l'objet et la pointe est inférieure à la demi-longueur d'onde (typiquement $200 \mathrm{~nm}$ ) celle-ci pénètre dans la zone du champ proche et le signal STOM apparaît. Il augmente rapidement (exponentiellement dans le cas idéal) jusqu'à ce que la pointe entre en contact avec l'objet.

Etape 2 : Lobjet continue sa course vers la fibre entraînant avec lui le microlevier. La variation de la distance microlevier-fibre va à son tour engendrer un deuxième système de franges dont la phase relative conduira à la connaissance du déplacement du microlevier par rapport à la fibre : Le faisceau STOM subira les mêmes réflexions que le faisceau topographique et présentera donc des franges parasites. Celles-ci pourront être éliminées ou tout du moins réduites en jouant sur les indices respectifs ou encore en introduisant un liquide adaptateur de même indice que celui du microlevier entre celui-ci et la fibre.

Signalons que, bien que la diffraction du champ évanescent soit pratiquement omnidirectionnelle, le calcul ne prendra en compte que des rayons normaux à la surface du microlevier. Cette approximation est justifiée par le fait que seuls les rayons paraxiaux seront transmis par la fibre monomode.

La différence d'ordre de grandeurs entre les deux faisceaux nous conduit à développer soit une détection STOM - AFM séquentielle, soit à compenser les intensités au moyen d'un filtre neutre.

2.3 DÉVELOPPEMENT EXPÉRIMENTALET RÉSULTATS. - Le montage expérimental est décrit sur la figure 4. Le positionnement de la fibre optique face à la pointe du microlevier est effectué à l'aide d'une platine de translation $(x y)$ piézoélectrique. Le balayage $x y z$ de l'objet est réalisé à l'aide d'un translateur piézoélectrique. Le faisceau topographique est issu d'un laser $\mathrm{He}-\mathrm{Ne}$ de longueur d'onde $\lambda=632,8 \mathrm{~nm}$. Il est modulé en amplitude au moyen d'une cellule acoustooptique puis injecté à l'aide d'une lentille dans la fibre qui l'acheminera vers le microlevier. Le signal de retour suit le trajet opposé, un cube séparateur est alors utilisé pour le diriger vers le détecteur. Le faisceau STOM, quant à lui, est issu d'une diode laser de longueur d'onde $\lambda=670$ $\mathrm{nm}$ modulable en amplitude à une fréquence différente du précédent. Il subit une réflexion totale sur l'objet engendrant ainsi le champ évanescent. La pointe du microlevier excitée par ce dernier va réémettre dans tout l'espace et donc en particulier en direction de la fibre qui acheminera le faisceau résultant vers le même détecteur.

Le résultat expérimental de l'approche en $z$ est représenté sur la figure 5 . Il confirme la prédiction théorique.

Plusieurs difficultés d'ordre pratique sont à souligner. Tout d'abord le faible rapport signal sur bruit du signal STOM dû tout d'abord au faible niveau du signal détecté et également aux multiples réflexions de la lumière sur la face d'entrée de la fibre et sur les autres composants optiques (lentilles, cube séparateur...). Ce problème a été résolu par l'utilisation d'un polariseur placé à l'entrée du photomultiplicateur. Le faisceau He-Ne étant polarisé au départ, le trajet d'allerretour dans la fibre introduit une rotation de cette polarisation qui n'affectera pas la lumière parasite. Le rapport signal sur bruit passe ainsi de 1 à 1000 .

D'autre part, le positionnement de la fibre au-dessus de la pointe est particulièrement critique (Fig. 6). Pour l'ajuster, la pointe est tout d'abord amenée en contact avec l'objet. La surface au voisinage de la pointe est alors éclairée avec le faisceau issu de la fibre (jouant le rôle d'émetteur et de collecteur). Afin de générer une image bidimensionnelle, la fibre balaye le plan $x y$ au moyen d'un actionneur piézoélectrique. La figure 7a est le résultat de ce balayage. Les franges sont dues 


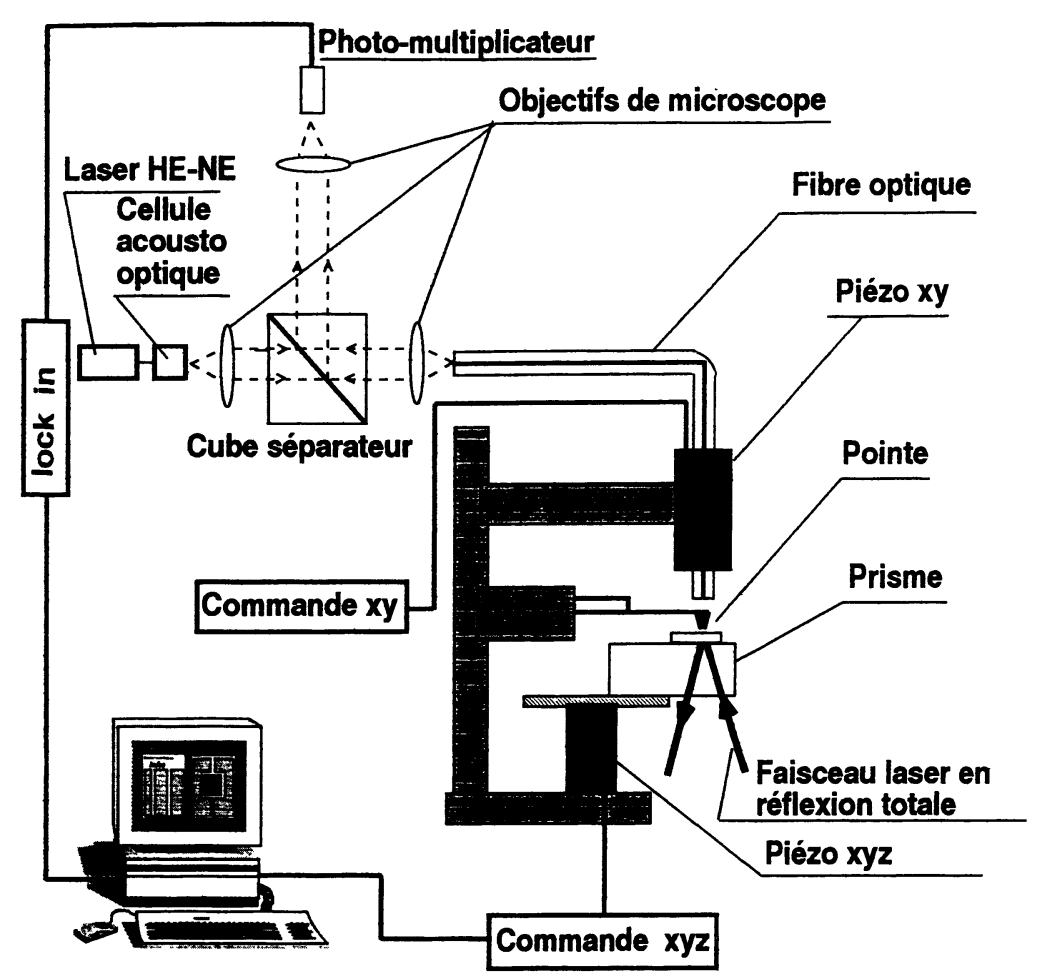

Fig. 4. - Schéma du montage expérimental du STOM - AFM.

[Experimental diagram of the AFM - STOM microscope.]
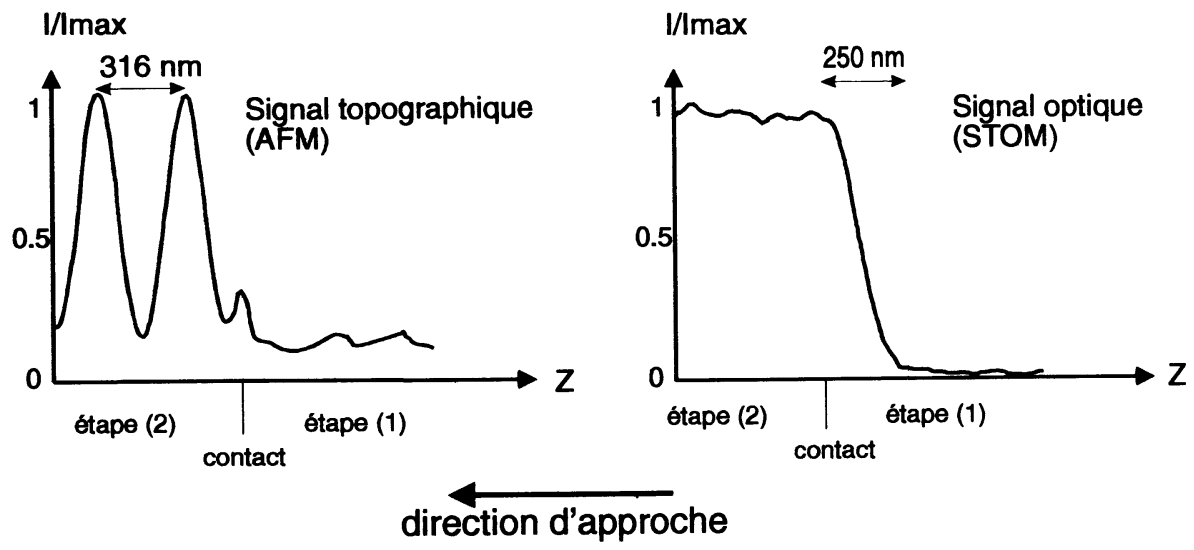

Fig. 5. - Courbes expérimentales correspondant à l'approche en $Z$. (Topographique à gauche et STOM à droite).

[Experimental approach curves. On the left, topographic signal, on the right, STOM signal.]

à des effets de coin d'air entre, d'une part la fibre et le microlevier et d'autre part, entre la fibre et l'objet. Cette cartographie, même grossière, nous permet de situer la position de la pointe par 

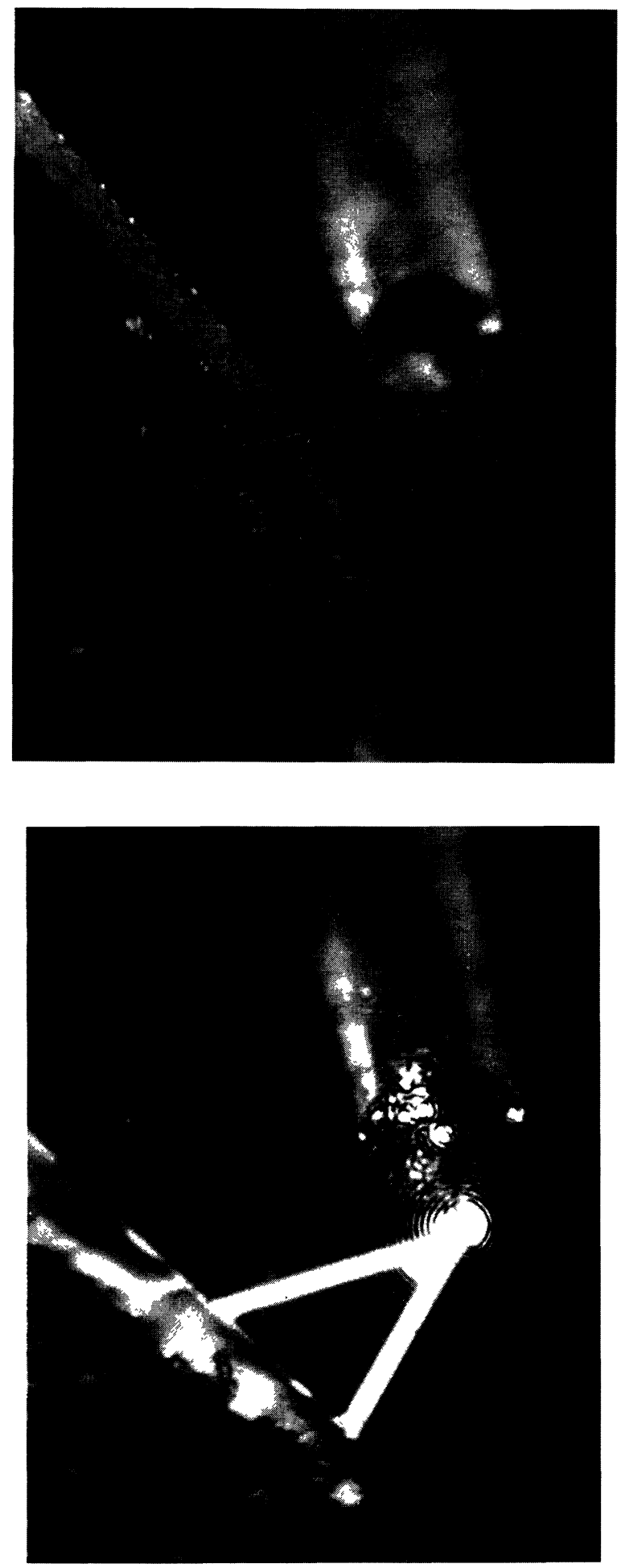

Fig. 6. - Positionnement de la fibre face au microlevier (longueur du microlevier : $200 \mu \mathrm{m}$, diamètre du coeur de la fibre : $5 \mu \mathrm{m}$ ).

[Photograph of the positioning of the fiber (length of the cantilever: $200 \mu \mathrm{m}$, diameter of the core of the fiber: $5 \mu \mathrm{m})$.] 


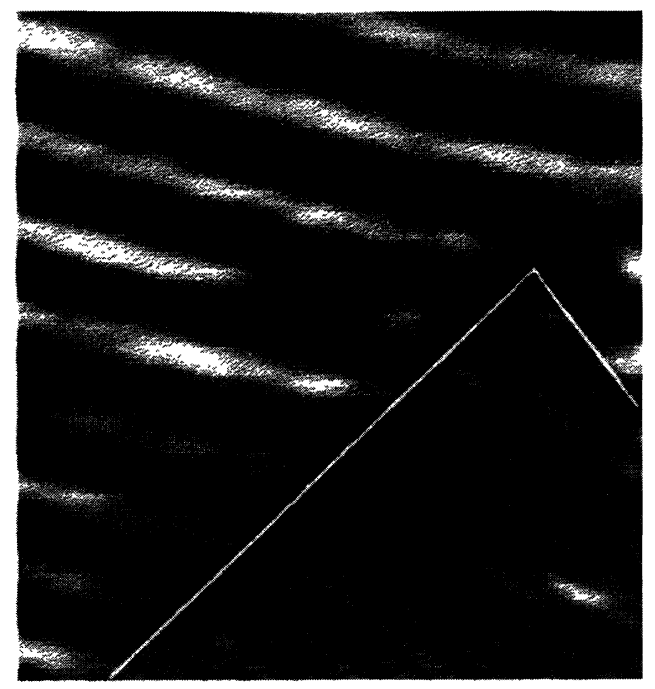

a)
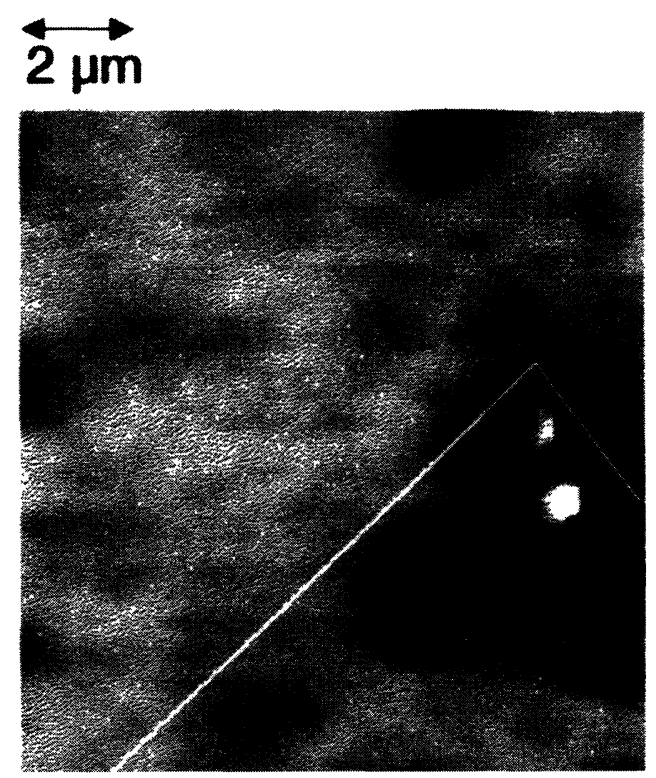

b)

Fig. 7. - Images de l'extrémité du microlevier. Celles-ci ont été obtenues par balayage du plan $x y$ au moyen de la fibre. a) la fibre éclaire l'ensemble microlevier-objet et capte la lumière réfléchie, b) la fibre capte le champ frustré par la pointe associée au microlevier).

[Images of the extremity of the cantilever. a) the fiber illuminates both the cantilever and the object and intercepts the reflected light, b) the fiber collects the diffracted beam coming from the tip.]

rapport à la fibre. Dans une deuxième expérience (Fig. 7b) nous avons dressé une cartographie de l'iniensité lumineuse diffractée par la pointe immergée dans le champ évanescent, en détectant le signal STOM avec la fibre optique jouant alors le rôle de collecteur. On constate l'apparition de deux foyers de lumière. Cette image, parfaitement reproductible, montre la complexité de l'interaction à l'intérieur de la pointe à géométrie pyramidale. On note ici la nécessité de réaliser 
une étude approfondie sur la distribution du champ au-dessus du microlevier. Cette étude est en cours de développement tant sur le plan expérimental que théorique.
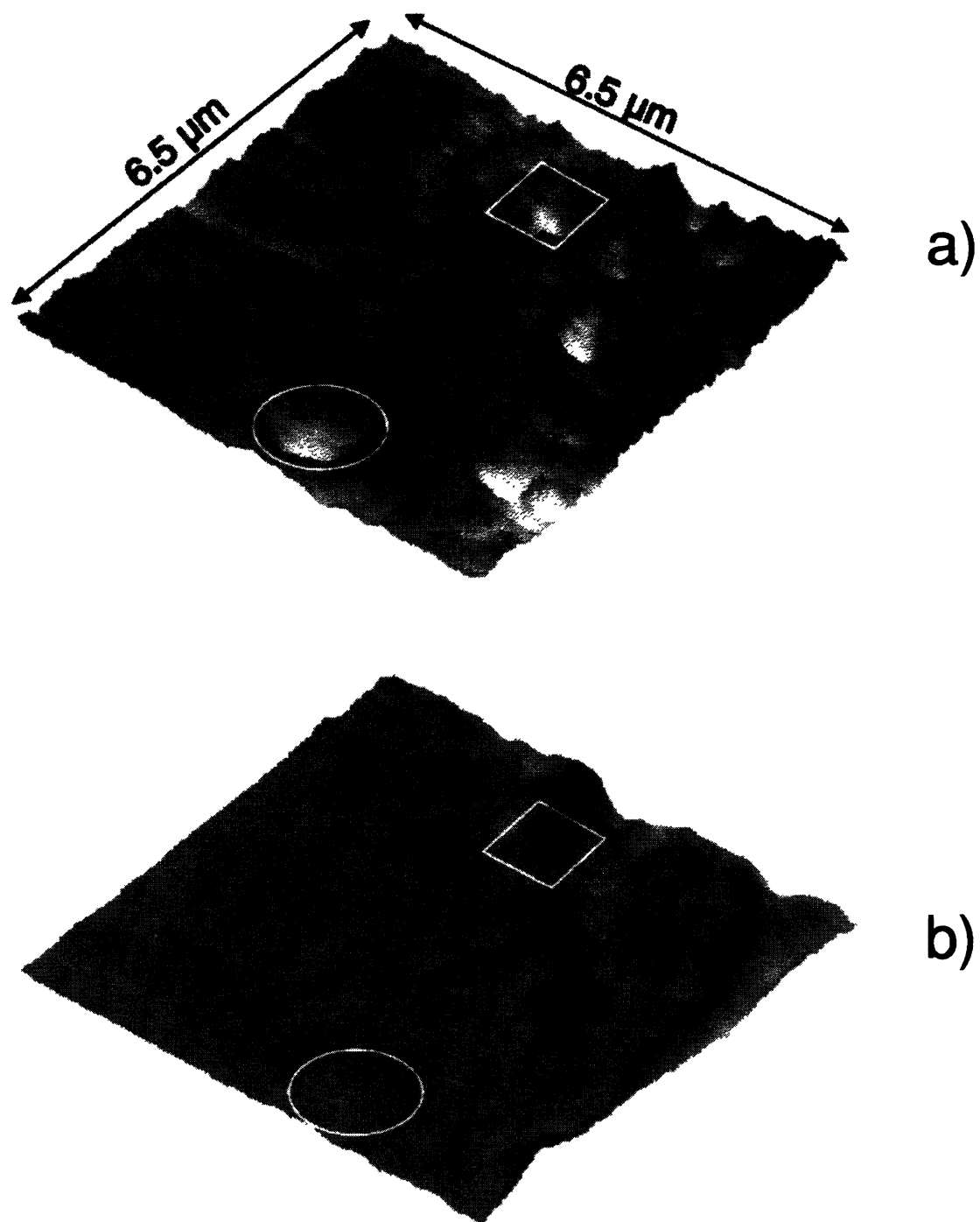

b)

Fig. 8. - Images topographique a) et optique b) d'une surface de verre attaquée à l'acide fluorhydrique. Les images sont partiellement corrélées. Certaines régions sont plus fidèlement reproduites (voir parties encadrées).

[Top view of topographic a) and STOM (b) images of a piece of glass chemically etched by HF. The images are partially correlated. Some regions (in the cercle and in the square) exhibit similarities.]

La figure 8 montre deux images d'une surface de verre attaqué à l'acide fluorhydrique. Limage topographique en a), bien résolue, présente les structures en cuillère caractéristiques de l'attaque chimique de la silice par ce type d'acide, alors que l'image optique en b), bien que moins résolue, présente dans son aspect général, une ressemblance certaine avec l'image topographique. 
Certaines régions montrent des similitudes beaucoup plus marquées (zone encerclée) alors que d'autres présentent une inversion de contraste (zone encadrée) due certainement à une variation d'indice local.

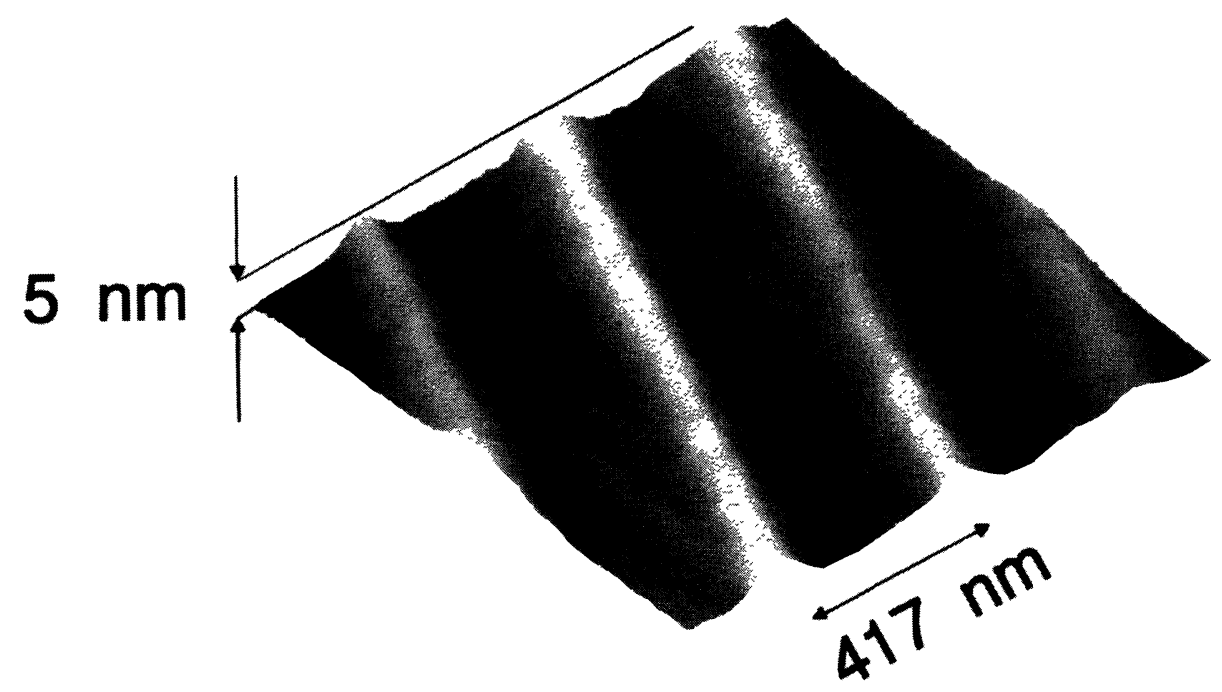

a)

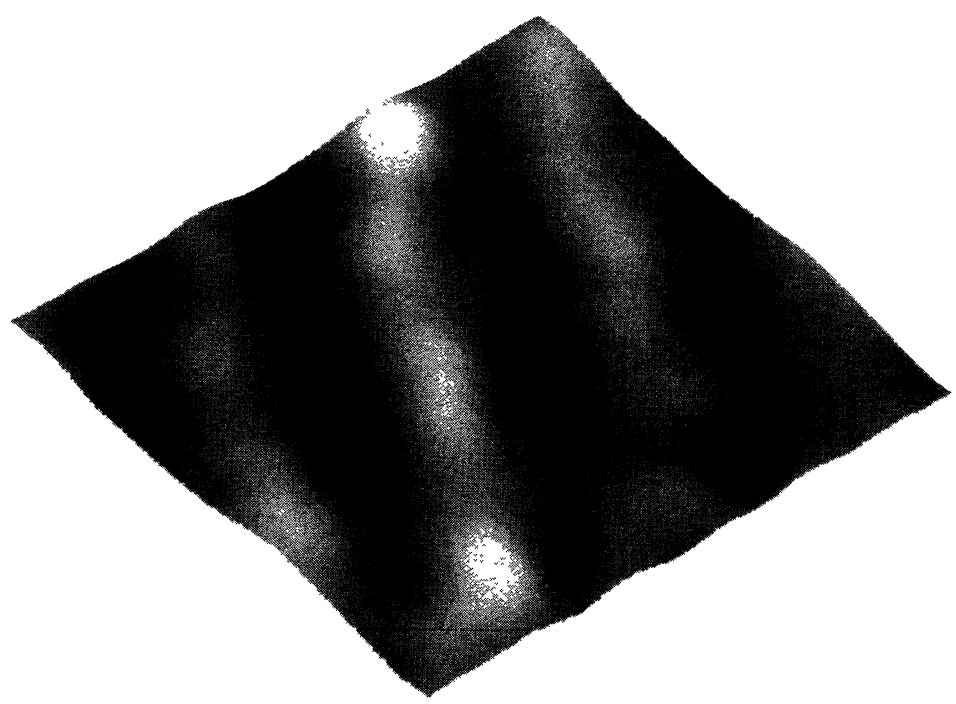

b)

Fig. 9. - Images topographique a) et optique b) d'un réseau de $417 \mathrm{~nm}$ de période et de $5 \mathrm{~nm}$ d'amplitude de modulation.

[Topographic image a) and STOM image b) of a very low profile grating.]

Un réseau de période $417 \mathrm{~nm}$ et de profondeur de modulation de $5 \mathrm{~nm}$ a été également analysé. La figure 9 montre les deux images topographique a) et STOM b) enregistrées l'une après l'autre. 
On note l'inversion de contraste entre les deux images, due à la différence des chemins optiques entre les deux faisceaux.

\section{Conclusion et perspectives.}

Lutilisation des pointes AFM en optique en champ proche présente de nombreux avantages sur l'utilisation des fibres optiques. D'une part, elles permettent l'examen de la distribution du champ optique sur la surface de l'objet sans risque de destruction de la sonde et de l'objet. D'autre part, elles sont parfaitement compatibles, par nature, avec la technologie AFM. La combinaison AFM - STOM est donc particulièrement simple. La résolution des images optiques dépendra essentiellement de la taille de la partie de la pointe qui entre dans le processus de diffraction (conversion de l'onde évanescente non homogène en onde progressive). Il est clair que l'usage d'une pointe AFM classique ayant une forme pyramidale avec un angle au sommet de 80 ou de $90^{\circ}$ ne constitue pas la solution optimale. En effet, la profondeur de pénétration moyenne du champ évanescent étant d'environ $100 \mathrm{~nm}$, la section utile de détection de la pointe est du même ordre de grandeur. Une solution déjà proposée [10] consistant en l'utilisation d'une pointe AFM classique à l'extrémité de laquelle on fait croître un composé carboné par "cracking" de molécules organiques sous microscope électronique à balayage (SEM), a déjà donné d'excellents résultats. Il a pu être montré que sur certains échantillons (oxyde d'indium-étain), la résolution optique dépasse $50 \mathrm{~nm}$ et conduit donc à des dispositifs aussi performants que les meilleurs microscopes optiques en champ proche actuels.

Ce travail a été effectué dans le cadre du programme interdisciplinaire ULTIMATECH/CNRS, France.

\section{References}

[1] Pohl D.W., Denk W., Lanz M., Appl. Phys. Lett. 44 (1984) 651-653.

[2] Porte L., Courjon D., Spectra 2000164 (1992) 40-48.

[3] Courjon D., Bainier C., Spajer M., J. Vac. Sci. Technol. B10 (1992) 2436-2439.

[4] Van Hulst N.F., Moers M.P.H., Noordman O.F.J., Faulkner T., Segerink F.B., Van Der Werf K., De Grooth B., Bögler B., SPIE Proceedings, Scanning probe microsc. 1639 (1992) 36-43.

[5] Toledo-Crow R., Yang P.C., Chen Y., Vaez-Iravani M., Appl. Phys. Lett. 60 (1992) 2957-2959.

[6] Baida F., Courjon D., Tribillon G., NFO Proc. ser. E 242 (1992) 71-78.

[7] Vigoureux J.M., J.O.S.A. A8 (1991) 1697-1701.

[8] Nazar F.M., Short notes J.P.N. J. Appl. Phys. 18 (1979) 1181-1182.

[9] Johnson P.B., Christy R.W., Phys. Rev. B6 (1972) 4370-4379.

[10] Moers M.P.H., Tack R.G., Noordman O.F.J., Segerink F.B., Van Hulst N.F., Bölger B., NFO Proc. ser. E 242 (1992) 79-86. 